\title{
COMMENT
}

\section{The future of faecal transplants}

\author{
Edward M. Giles ${ }^{1,2}$, Gemma L. D’Adamo ${ }^{1,3}$ and Samuel C. Forster ${ }^{1,3 *}$ \\ Faecal microbiota transplant (FMT) is now accepted as an effective treatment for Clostridioides \\ difficile infections. With the increasing number of FMT treatments and clinical trials for other \\ indications there is an urgent need for standardized regulations to ensure patient safety and \\ focused development of safer, rationally designed, microbiota-based medicines.
}

Randomized controlled trials of faecal microbiota transplant (FMT) for treatment of Clostridioides difficile infection have reported over $90 \%$ efficacy, largely irrespective of preparation and administration strategy. Subsequent clinical trials suggest FMT as an effective treatment option for other conditions, such as ulcerative colitis. Despite the non-specific nature of the treatment, FMT has shown a remarkably low rate of serious adverse events. Although current trials are typically focused on specific patient groups, the benefits of FMT in reducing the personal and economic impacts of disease are now undeniable.

Nevertheless, the transfer of live microorganisms from healthy donors to sick patients has inherent risks. The recent FMT containing multidrug-resistant bacteria that resulted in a patient death ${ }^{1}$, coupled with three previous reports of fatalities through aspiration ${ }^{2,3}$ and toxic megacolon ${ }^{4}$, illustrate some of these issues. The risk of infection, although small, depends on the FMT sample and is possibly higher in patients with disturbed microbiota, for example, after antibiotic treatment. Furthermore, FMT-derived microorganisms might also be transmitted further and, although the risk and impacts are unclear, this should be considered, in particular in healthcare facilities.

Although efforts to reach consensus are progressing $^{5,6}$, regulatory standards are limited or absent in many jurisdictions. Although omitted in the recently reported death, the use of standard microbiological screening should mitigate the risk of transferring known disease-causing microorganisms, particularly those carrying antimicrobial resistance. In addition, many gut microorganisms, most notably Fusobacterium nucleatum and Sutterella species, have been linked to various diseases and conditions from inflammatory bowel disease to cancer. The specific links are not well understood, but likely depend on genetics, immune state and environmental factors. Incorporating appropriate screening strategies for these microorganisms will be challenging. The capacity to safely deliver FMT to patients will depend on standardized, highly specialized laboratories for stool preparation. These laboratories will require expertise in collection and management of donor medical and lifestyle data; appropriate sample collection, preparation and storage procedures; thorough screening of donor material; and standardized methods for reporting adverse events

Another challenge is identifying conditions for which microbiota-based medicines have true clinical potential. Well-designed clinical trials of FMT will help to validate the role of the microbiota in disease and to identify the specific bacteria or metabolites responsible for this effect. These trials could also guide the development of targeted bacteriotherapy. For some conditions, a targeted or even personalized approach may well achieve the best and safest therapeutic outcomes for patients.

Due to the complexity and variability in donor stools, coupled with our limited understanding of the ecological forces that shape the microbiota, FMT will always present a risk for some patients. For these reasons, next-generation microbiota-based medicines will likely become the preferred option. These defined interventions with rationally selected mixtures of microorganisms or their products are more amenable to rigorous clinical trials. These therapies will provide the ability to determine optimal treatment regimens and to define risk profiles that can be more reliably managed than FMT.

\footnotetext{
FDA. Important Safety Alert Regarding Use of Fecal Microbiota for Transplantation and Risk of Serious Adverse Reactions Due to Transmission of Multi-Drug Resistant Organisms. Fda.gov https://www.fda.gov/vaccines-blood-biologics/safety-availability biologics/important-safety-alert-regarding-use-fecal-microbiotatransplantation-and-risk-serious-adverse (2019).

2. Baxter, M. et al. Fatal aspiration pneumonia as a complication of fecal microbiota transplant. Clin. Infect. Dis. 61, 136-137 (2015).

3. Kelly, C. R. et al. Fecal microbiota transplant for treatment of Clostridium difficile infection in immunocompromised patients. Am. J. Gastroenterol. 109, 1065-1071 (2014).

4. Solari, P. R. et al. Tempered enthusiasm for fecal transplant. Clin. Infect. Dis. 59, 319 (2014).

5. Cammarota, G. et al. European consensus conference on faecal microbiota transplantation in clinical practice. Gut 66, 569 (2017).

6. Mullish, B. H. et al. The use of faecal microbiota transplant as treatment for recurrent or refractory Clostridium difficile infection and other potential indications: joint British Society of Gastroenterology (BSG) and Healthcare Infection Society (HIS) guidelines. Gut 67, 1920-1941 (2018).
} 